\title{
Internações em Unidade de Atenção Psicossocial: análise antes e durante a pandemia por COVID-19
}

\author{
Psychosocial Care Unit hospitalizations: analysis before and during the COVID-19 \\ pandemic
}
Hospitalizaciones a una Unidad de Atención Psicosocial: análisis antes y durante la pandemia de COVID-19

Bruna Caroline Ruppelt ${ }^{1 *}$, Alexandra do Nascimento Damasio Flores ${ }^{1}$, Valquíria Toledo Souto ${ }^{2}$, Maria Denise Schimith ${ }^{1}$, Sidney da Silva Marques ${ }^{3}$, Etiane de Oliveira Freitas ${ }^{1}$, Marlene Gomes Terra$^{1}$, Márcia Aparecida Ferreira de Oliveira ${ }^{4}$, Daiana Foggiato de Siqueira ${ }^{1}$.

\section{RESUMO}

Objetivo: Analisar as internações de pessoas com problemas de saúde mental em uma Unidade de Atenção Psicossocial de um hospital universitário antes e durante a pandemia por COVID-19. Métodos: Estudo transversal com base em dados secundários obtidos por meio do serviço de estatística de um Hospital Universitário do interior do Estado do Rio Grande do Sul. Resultados: A análise de 308 (100\%) internações ocorridas no período de abril a outubro de 2019 e de abril a outubro de 2020 identificou que em ambos os períodos houve prevalência de internações de mulheres brancas. No período de pandemia, o número de transtornos depressivos aumentou, quando comparado ao período anterior. Conclusão: Os resultados dessa pesquisa demonstraram que a pandemia causada pelo coronavírus impacta nos serviços de saúde mental, inclusive nos serviços de internação, uma vez que medidas necessárias para enfrentar a situação de emergência acabaram por influenciar no funcionamento desses. Assim, é necessária a defesa e priorização dos serviços de saúde mental, sobretudo no contexto atual.

Palavras-chave: Enfermagem, Hospitalização, Saúde mental, Serviços de saúde, Transtornos mentais.

\begin{abstract}
Objective: To analyze the admissions of people with mental health problems in a Psychosocial Care Unit of a university hospital before and during the COVID-19 pandemic. Methods: Cross-sectional study based on secondary data obtained through the statistical service of a University Hospital in the interior of the state of Rio Grande do Sul. Results: The analysis of $308(100 \%)$ admissions that occurred from April to October 2019 and April to October 2020 identified that in both periods there was a prevalence of hospitalizations of white women. During the pandemic period, the number of depressive disorders increased compared to the previous period. Conclusion: The results of this research showed that the pandemic caused by the coronavirus impacts on mental health services, including inpatient services, since the necessary measures to face the emergency situation ended up influencing their functioning. Thus, it is necessary to defend and prioritize mental health services, especially in the current context.
\end{abstract}

Keywords: Nursing, Hospitalization, Mental health, Health services, Mental problems.

\section{RESUMEN}

Objetivo: Analizar los ingresos de personas con problemas de salud mental en una Unidad de Atención Psicosocial de un hospital universitario antes y durante la pandemia de COVID-19. Métodos: Estudio transversal a partir de datos secundarios obtenidos a través del servicio estadístico de un Hospital Universitario del interior del estado de Rio Grande do Sul. Resultados: Análisis de 308 (100\%) ingresos ocurridos a partir de abril a octubre de 2019 y de abril a octubre de 2020 identificaron que en ambos períodos hubo una prevalencia de hospitalizaciones de mujeres blancas. Durante el período pandémico, el número de

\footnotetext{
1 Universidade Federal de Santa Maria (UFSM), Santa Maria - RS. *E-mail: ruppeltbruna@gmail.com

2 Hospital SOS Cárdio, Florianópolis - SC.

3 Hospital Universitário de Santa Maria, Santa Maria - RS.

4 Universidade de São Paulo (USP), São Paulo - SP.
} 
trastornos depresivos aumentó en comparación con el período anterior. Conclusión: Los resultados de esta investigación arrojaron que la pandemia provocada por el coronavirus impacta en los servicios de salud mental, incluidos los servicios de internación, ya que las medidas necesarias para enfrentar la situación de emergencia terminaron influyendo en su funcionamiento. Por tanto, es necesario defender y priorizar los servicios de salud mental, especialmente en el contexto actual.

Palabras clave: Enfermería, Hospitalización, Salud mental, Servicios de salud, Transtornos mentais.

\section{INTRODUÇÃO}

A Política Nacional de Saúde Mental, alinhada com as diretrizes da Reforma Psiquiátrica Brasileira (Lei 10.216/2001), impulsionou a construção de um modelo humanizado de cuidado em saúde mental (BRASIL, 2001). Essa Política tem como objetivo reduzir de forma gradativa os leitos de internação psiquiátrica e aumentar os serviços e dispositivos extra hospitalares, permitindo o cuidado integral em saúde mental com ênfase na liberdade e no exercício da cidadania (BRASIL, 2001; AMARANTE P e NUNES MO, 2018).

Embora os demais dispositivos da Rede de Atenção Psicossocial (RAPS), como os Centro de Atenção Psicossocial, ambulatório especializado e Unidade de Acolhimento sejam eficazes e a internação seja indicada como último recurso terapêutico, percebe-se que no Brasil a sua prática se faz muito presente como tratamento inicial. Essa prática está na contramão do preconizado pela Política Nacional de Saúde Mental e pela Reforma Psiquiátrica Brasileira (ZANARDO GLP, et al., 2017; ZANARDO GLP, et al., 2018).

Essa discussão se faz necessária neste momento em que a área da saúde mental tem ganhado maior visibilidade entre a população, sobretudo em decorrência da pandemia de COVID-19 e suas múltiplas implicações psicossociais na vida das pessoas. Segundo dados epidemiológicos oriundos de um estudo realizado no Brasil, nesse período os indivíduos sentiram-se com frequência, deprimidos, tristes, ansiosos, com insônia (FARO A, et al., 2020).

Segundo pesquisa realizada entre os meses de março e abril de 2020 , com mais de 1000 pessoas em 23 estados brasileiros, os problemas de saúde mental aumentaram de forma preocupante nesse atual contexto. Os casos de ansiedade e estresse aumentaram cerca de $80 \%$ na população, enquanto os casos de depressão praticamente dobraram. A mesma pesquisa também constatou que as mulheres são mais suscetíveis a sofrerem com estresse e ansiedade durante este período (DUARTE MQ, et al., 2020). Assim, há um número expressivo de pessoas que precisam de atendimento em saúde mental, nos diversos dispositivos da RAPS, incluindo a internação psiquiátrica (ORNELL F, et al., 2020).

Desse modo, justifica-se a realização desse estudo pela possibilidade de levantar as características das internações ocorridas neste período, uma vez que o município em questão, não conta com Centro de Atenção Psicossocial de acolhimento em turno integral, também a fim de incitar reflexões acerca da temática em tempos de pandemia e colaborar com a Política de Saúde Mental, com estudiosos da área, com a comunidade acadêmica, com os usuários e com a sociedade em geral. O presente estudo teve como objetivo analisar as internações de pessoas com problemas de saúde mental de uma Unidade de Atenção Psicossocial de um hospital universitário antes e durante a pandemia por COVID-19.

\section{MÉTODOS}

Trata-se de um estudo transversal a partir de dados secundários disponibilizados pelo serviço de estatística de um Hospital Universitário do interior do Estado do Rio Grande do Sul (RS) acerca de internações hospitalares em Unidade de Atenção Psicossocial (UAP) dos períodos de abril a outubro de 2019 (período anterior a pandemia) e de abril a outubro de 2020 (decorrer da pandemia). Em contexto de normalidade, a unidade dispõe de 30 leitos para pacientes com transtornos psíquicos graves, distribuídos em duas enfermarias femininas, duas masculinas e dois apartamentos de dois leitos para casos de comorbidades clínicas. No entanto, no período de pandemia pela COVID-19, a partir de março de 2020 o Comitê de Crise da instituição adotou medidas preventivas que resultaram na redução da oferta de leitos. 
Com base nas informações disponíveis pelo serviço, os pesquisadores desenvolveram um instrumento para sistematizar a coleta de dados; composto pelas seguintes variáveis: sexo, idade, raça/cor, procedência, período de internação, tempo de internação e diagnóstico. Os dados foram duplamente digitados em planilhas Excel (2012) e inconsistências, corrigidas.

A análise dos dados foi realizada no programa Statistical Package for Social Sciences (SPSS) for Windows, versão 19. As variáveis categóricas foram apresentadas em frequências simples ( $n$ ) e relativas (\%). As variáveis contínuas foram expressas em medidas de tendência central (valor mínimo, valor máximo e média) e medidas de dispersão (desvio padrão). Para verificar a diferença entre as variáveis estudadas, antes e após a pandemia, foram utilizados os testes $T$ de Student e o Qui quadrado. Os resultados foram considerados estatisticamente significativos se $\mathrm{p}<0,05$.

Foram respeitados todos os aspectos éticos, porém, por ser pesquisa com dados secundários tem-se a dispensa do Termo de Consentimento Livre e Esclarecido, bem como da apreciação pelo Comitê de Ética em Pesquisa conforme estabelecido pela Resolução 510/2016, art. 1 , inciso V.

\section{RESULTADOS}

Foram analisadas $308(100 \%)$ internações ocorridas na Unidade de Atenção Psicossocial no período. Dessas, 165(53,6\%) ocorreram antes da pandemia (2019) e $143(46,4 \%)$ durante a pandemia (2020). Sobre as características dessas internações, tem-se que em ambos os anos houve predomínio de internações de mulheres brancas e procedentes do município de Santa Maria. Observa-se que a média do tempo de internação de 2020 foi inferior à média de 2019 ( $p<0,05)$, já a média de idade não diferiu entre os anos (Tabela 1).

Tabela 1 - Caracterização das internações quanto à idade, sexo, raça/cor, cidade de procedência e tempo de internação nos anos de 2019 e 2020.

\begin{tabular}{lccc}
\hline Variáveis & $\begin{array}{c}\text { Antes da pandemia (2019) } \\
\mathbf{N}(\%)\end{array}$ & $\begin{array}{c}\text { Durante a pandemia (2020) } \\
\mathbf{N}(\%)\end{array}$ & $\boldsymbol{p}$ \\
\hline Número de Internações & $165(53,6)$ & $143(46,4)$ & \\
Idade (Média) & $41,03 \pm 14,85$ & $40,31 \pm 13,57$ & 0,66 \\
\hline Sexo & & & 0,38 \\
\hline Masculino & $68(41,2)$ & $66(46,2)$ & \\
Feminino & $97(58,8)$ & $77(53,8)$ & 0,10 \\
\hline Raça/Cor & & & \\
\hline Branca & $151(91,5)$ & $120(83,9)$ & \\
Preta & $7(4,2) 4$ & $15(10,5)$ & 0 \\
Parda & $2(1,2)$ & & 0,06 \\
\hline Cidade & & $93(65,0)$ & \\
\hline Santa Maria & $106(64,2)$ & $50(35,0)$ & \\
Outras & $59(35,8)$ & $13,48 \pm 8,40$ & $0,00^{*}$ \\
\hline Tempo de internação (no dias) & $20,89 \pm 16,74$ & & \\
\hline
\end{tabular}

Legenda: ${ }^{*} p<0,05$.

Fonte: Ruppelt BC, et al., 2021.

Está sendo apresentada a distribuição das internações do mês de abril a outubro dos anos de 2019 e 2020. Conforme observado percebe-se que em 2019 (antes da pandemia) houve um maior número de internações nos meses de maio e agosto, enquanto em 2020 (durante a pandemia) o maior número de internação foi em outubro (Figura 1). 
Figura 1 - Distribuição das internações de acordo com o mês.

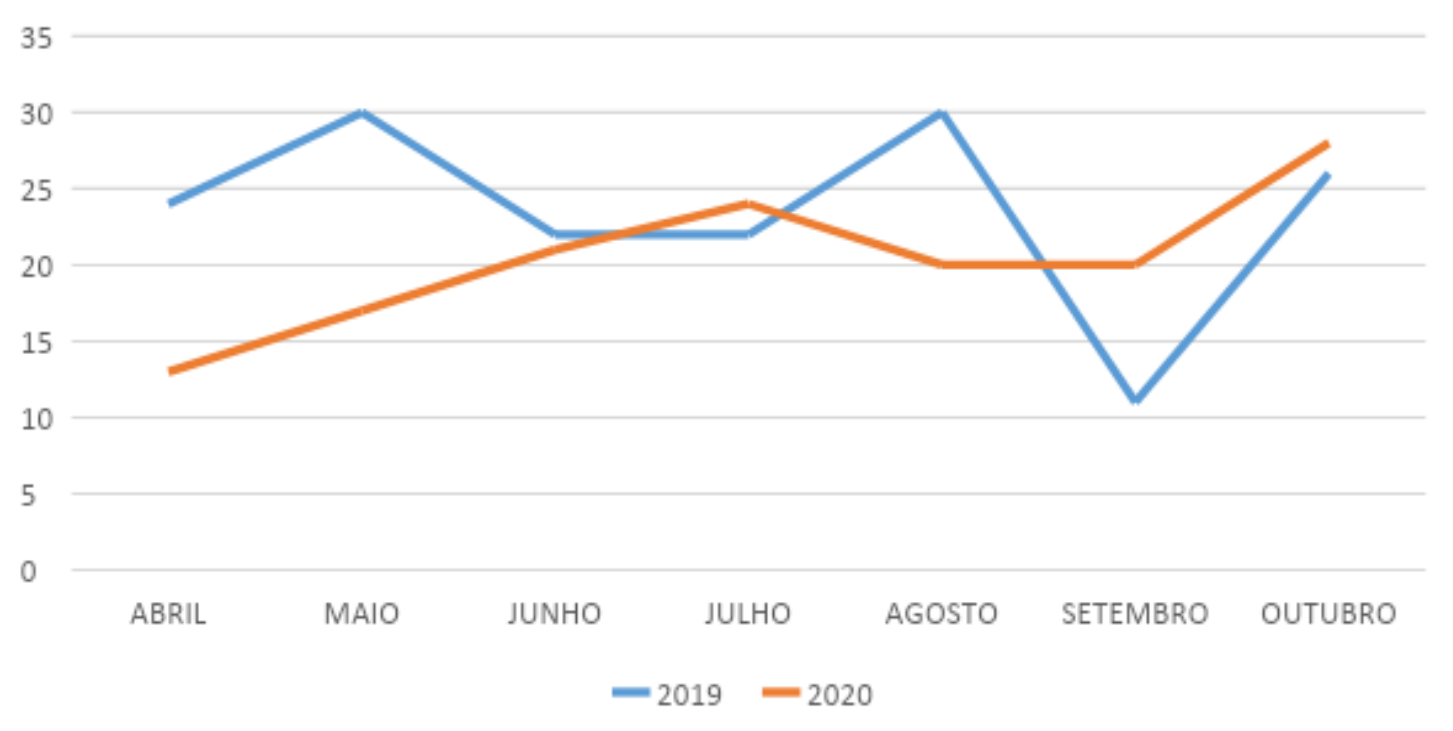

Fonte: Ruppelt BC, et al., 2021.

Em relação ao diagnóstico dos usuários internados na Unidade de Atenção Psicossocial têm-se como prevalente no ano de 2019 (antes da pandemia) o "Transtorno Bipolar e Transtornos Relacionados" e em 2020 (durante a pandemia) o diagnóstico "Espectro da Esquizofrenia e Outros Transtornos Psicóticos" (Tabela 2).

Tabela 2 - Caracterização das internações quanto ao diagnóstico segundo o Manual Diagnóstico E Estatístico de Transtornos Mentais 5a Edição (DSM-5) das internações nos anos de 2019 e 2020.

\begin{tabular}{lcc}
\hline Classificação DSM-5 & $\begin{array}{c}\mathbf{2 0 1 9} \text { (antes da } \\
\text { pandemia) \% (n) }\end{array}$ & $\begin{array}{c}\mathbf{2 0 2 0} \text { (durante a } \\
\text { pandemia) \% (n) }\end{array}$ \\
\hline Espectro da Esquizofrenia e Outros Transtornos Psicóticos & $29,9(36)$ & $32,6(47)$ \\
Transtorno Bipolar e Transtornos Relacionados & $32,1(54)$ & $30,6(44)$ \\
Transtornos Depressivos & $18,1(41)$ & $21,6(30)$ \\
Transtornos da Personalidade & $9,2(16)$ & $9,7(14)$ \\
Transtornos do Neurodesenvolvimento e Transtornos & $7,1(12)$ & $5,5(8)$ \\
Neurocognitivos & $1,2(2)$ & --- \\
Transtorno Mental Não Especificado & $2,4(4)$ & --- \\
Outras condições & & \\
\hline
\end{tabular}

Fonte: Ruppelt BC, et al., 2021.

\section{DISCUSSÃO}

Os resultados da pesquisa mostram que, no ano de 2019 o número de internações na UAP foi maior quando comparado ao mesmo período em 2020. Sabe-se que, alterações nos serviços de saúde ocorreram devido ao contexto de pandemia por COVID-19, sendo assim, os serviços de saúde mental também foram afetados. A Organização Mundial da Saúde (OMS) concluiu que, neste período, mais de um terço (35\%) dos serviços de saúde mental tiveram interrupções nas intervenções de emergência, ou seja, situações graves nas quais os usuários apresentaram delírios, convulsão e síndrome de abstinência devido ao uso abusivo de substâncias psicoativas (UNITED NATIONS, 2020).

Não obstante, uma série de medidas preventivas orientadas pelo Comitê de Crise ocorreu na UAP, o que resultou na redução por 60 dias da oferta de leitos, de 30 para 20 vagas. Outra medida adotada foi em relação 
à admissão dos usuários, mais rigorosa, visto que se estes apresentassem sintomas suspeitos de COVID-19, deveriam aguardar em isolamento, e após este período a internação na UAP poderia ocorrer. Com isso, houve uma limitação e redução das vagas disponíveis na unidade, o que pode ter implicado também na situação de redução de internações neste período (EMPRESA BRASILEIRA DE SERVIÇOS HOSPITALARES (EBSERH), 2020).

Diferentes medidas frente ao contexto de pandemia foram adotadas em todas as regiões do mundo. Documento elaborado pela Rede Italiana de Segurança em Saúde apresenta recomendações a serem adotadas em instituições psiquiátricas hospitalares e residenciais, como: o fechamento de ambulatórios e centros dia ou de semi-residência; suspensão de visitas de familiares e encaminhamento de pacientes menos graves internados para as famílias até ao final da emergência causada pela COVID-19 (LA REGINA M, et al., 2020). Todas estas restrições nos serviços citados implicam diretamente na assistência em saúde mental.

Diante dessa situação, há de se pensar que fenômenos relacionados à saúde psíquica serão ainda mais recorrentes no mundo pós-pandemia, impactando os serviços de saúde mental. Segundo estudo chinês, 35\% da população em geral vem apresentando sofrimento psíquico, enquanto o sistema não se encontra preparado para resolver essa demanda, sendo assim, a assistência em saúde mental deve receber ainda mais visibilidade, incentivo e fomento orçamentário para que atue no sofrimento agravado pela pandemia. Ainda, se faz necessário uma maior preparação e acolhimento dos profissionais atuantes nesta área, bem como estratégias que incentivem o uso da telessaúde a fim de manter vínculo com os usuários, bem como, para atender a demanda dos novos (QIU J, et al., 2020).

No Brasil, devido à alta demanda de leitos de terapia intensiva para pessoas com diagnóstico de COVID19, alguns leitos destinados aos usuários com transtornos mentais e até mesmo hospitais psiquiátricos foram transformados em enfermarias e leitos de terapia intensiva. Estratégia a fim de minimizar a situação de lotação nos serviços de saúde causada pelo coronavírus (NORONHA KVMS, et al., 2020).

No Rio Grande do Sul, o cenário também impulsionou a divulgação pela gestão estadual de manuais de conduta aos serviços, sugerindo aos serviços de saúde mental que os pacientes com sintomas leves de COVID-19 sejam avaliados para a possibilidade de internação domiciliar e com as recomendações necessárias aos familiares e/ou cuidadores (BRASIL, 2020a). Em situações de saúde em que não é possível ocorrer internação domiciliar, em virtude do quadro psíquico agravado ou devido à ausência de uma rede de apoio, é necessária a garantia do isolamento do usuário em quarto individual (BRASIL, 2020).

Desse modo, é possível compreender que os impactos das medidas tomadas nos serviços de saúde mental durante a emergência causada pelo coronavírus podem implicar na redução do número de internações ocorridas durante esse período. Ao refletir acerca da realidade do município em que se encontra a UAP, que dispõe de serviços extra-hospitalares na RAPS, é necessário que estes sejam potencializados neste período crítico a fim de amparar os usuários que demandam de cuidados em saúde mental e que possam ofertar cuidados de acolhimento em turno integral.

Nesse sentido, os dispositivos de base comunitária podem repensar o atendimento das pessoas com problemas de saúde mental, tendo uma continuidade no tratamento e também adaptando as atividades em grupo para que o vínculo não seja perdido. Algumas possibilidades estão em realizar atividades com um número menor de participantes e em mais dias, auxiliar de forma on-line aqueles em que o acesso às tecnologias é possível, promover visitas periódicas às residências dos usuários a fim de acolher a família, bem como, potencializar a adoção dos cuidados frente à pandemia.

Na variável a respeito do sexo, os resultados apontam que as mulheres internaram na UAP mais do que os homens, tanto em 2019, quanto em 2020. De acordo com o Ministério da Saúde, mulheres têm duas vezes mais chance de apresentar sofrimento psíquico do que os homens, o que está relacionado, principalmente, à diferença de gênero (BRASIL, 2013).

Nesse contexto, ao analisar os fatores que contribuem para os problemas de saúde mental prevalentes no sexo feminino, destacam-se os aspectos socioeconômicos e estruturais (FERREIRA OGL, et al., 2012). É de 
conhecimento social que diversas relações desiguais de gênero existem de forma a explicar e/ou aumentar essa maior suscetibilidade de transtornos mentais entre as mulheres. Nesta lógica, acredita-se que as atribuições femininas tradicionais como o papel de esposa, de mãe, de filha, de cuidadora, quando associados a fatores estruturais que envolvem desemprego, situação de violência e condições precárias de vida e saúde, contribuem para o adoecimento psíquico das mulheres (ZANELLO V, et al., 2015).

Esta condição pode ser ainda mais agravada em determinados contextos, como nos das cidades rurais. No Brasil, segundo estudos nacionais, a prevalência de transtornos mentais em mulheres de áreas urbanas varia de $22,1 \%$ a $34,2 \%$, enquanto as de áreas rurais a prevalência variou de $23,3 \%$ a $66,9 \%$ (MARTIN OS e MEUCCI RD, 2020).

Além disso, um estudo que analisou o perfil das internações psiquiátricas em um hospital geral da região Sul, aponta que o maior número de mulheres internadas tinha diagnóstico de transtorno depressivo $(46,4 \%)$, o que confirma que, além desse público adoecer de duas a três vezes mais por depressão, o adoecimento se torna grave sendo necessária a internação (BRAGÉ EG, et al., 2020). Dessa forma, compreende-se que os estudos evidenciam fatores que contribuem para um maior adoecimento da população feminina por transtornos mentais, sendo que estes também podem compilar para um maior número de internações psiquiátricas.

Outro resultado evidenciado no estudo está atrelado ao tempo de internação na UAP, visto que este difere

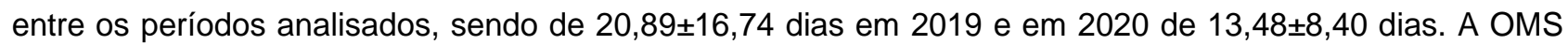
refere que $93 \%$ de 130 países pesquisados relataram mudanças e interrupções em um ou mais de seus serviços de Saúde Mental em 2020, o que impacta também nos serviços de internação, repercutindo no tempo de duração destas (UNITED NATIONS, 2020).

Diante disso, pode-se inferir que o número de dias de internação foi menor em 2020 devido às mudanças realizadas nos serviços de saúde, no que se refere a medidas de biossegurança e controle da disseminação de casos de COVID-19. Segundo o Plano de Contingência para Infecção Humana pelo novo coronavírus (COVID-19), elaborado pelo hospital universitário, profissionais tiveram que ser redimensionados, pela necessidade de equipes específicas na triagem de pacientes, além da expansão do número de leitos, o que também demanda recurso pessoal (EBSERH, 2020).

Outras medidas foram implementadas na UAP como as ações para otimização da gestão de leitos e ampliação da equipe de gestão de alta. Com isso, houve a revisão de internações com longa permanência (30 dias ou mais nas unidades de internação e 02 dias ou mais no pronto socorro). Devido a situação emergencial a equipe passou a restringir os cuidados de saúde mental ao estritamente necessário, visando a estabilização psíquica, minimizando o risco de infecção por COVID-19 em vista da menor permanência em ambiente hospitalar (EBSERH, 2020). Maior celeridade no tempo de internação pode ser vista sob uma perspectiva positiva em relação à Reforma Psiquiátrica e a orientação de que o tratamento ocorra, preferencialmente, em serviços territoriais (BRASIL, 2001).

Vale ressaltar que muitos usuários desconhecem a existência das demais possibilidades de tratamento em saúde mental, o que contribui para a procura por internação, o que por vezes não é o ideal em diversas situações. Entretanto, muitos realmente necessitam de acolhimento em turno integral, pois as demais tentativas de cuidados não foram exitosas. Isto reforça que, mesmo com a situação voltada para a pandemia, os serviços de saúde mental devem continuar funcionando e serem potencializados, a fim de manter o acolhimento das demandas, que se desamparadas podem ser agudizadas e requerer nova internação (PEREIRA AR e JOAZEIRO G, 2015).

Outra situação que impactou a rotina da UAP foi a suspensão das visitas e limitação de acompanhantes durante o período de pandemia, o que também contribui para que a internação fosse menos longa (EBSERH, 2020). Sabe-se que as pessoas com transtornos mentais necessitam de acolhimento, socialização, participação em atividades que estimulem sua cognição e afeto, o que acaba por ser limitado diante do contexto vivenciado. Neste sentido, ao mesmo tempo em que esta limitação influencia negativamente 0 cuidado das pessoas em sofrimento psíquico, estas devido a suas condições, sentem de forma mais intensa 
as medidas de isolamento social. Sendo assim, necessitam ainda mais de uma rede de apoio fortalecida, composta por familiares e amigos durante o processo de tratamento, o que infelizmente, não se faz mais possível de forma presencial no ambiente hospitalar devido ao contexto atual (BRASIL, 2013).

No que tange ao número de internações, os resultados apontam uma maior ocupação de leitos pelos procedentes do município de Santa Maria, cidade na qual a UAP está localizada. Isto se deve pelo fato de o número de leitos para assistência em saúde mental ser determinado pelo número de habitantes dos municípios. Segundo a portaria GM/MS, № 148/2012, a distribuição de leitos hospitalares para atenção psicossocial tem como parâmetro 1 leito para cada 23 mil habitantes. Dessa maneira, sabe-se que Santa Maria é a cidade mais populosa da 4 a Coordenadoria Regional de Saúde, o que faz com que tenha um maior número de leitos ocupados (BRASIL, 2016).

No presente estudo constatou-se, também, que as internações por transtornos depressivos representaram $21,6 \%$ do total durante a pandemia, enquanto no período anterior, o percentual era de $18,1 \%$. Sabe-se que o contexto pandêmico exigiu que muitas medidas nunca antes vivenciadas pela população fossem incluídas no cotidiano, destacando-se entre elas, o distanciamento social. Essa medida é necessária para diminuir a interação entre as pessoas em cenários onde existem a transmissão comunitária do vírus (BRASIL, 2020c).

Além das medidas de distanciamento, vivencia-se, o medo da contaminação, o medo de perder um familiar, os sentimentos de frustração, de solidão, de ansiedade, a falta de informações confiáveis sobre a doença e os cuidados frente a ela, além das perdas financeiras e o estigma da doença atingiram muitos indivíduos no mundo todo e de todas as faixas etárias (SCHMIDT B, et al., 2020; PEREIRA MD, et al., 2020). Dessa maneira, o impacto do distanciamento social contribuiu para que efeitos psicológicos negativos aumentassem na população e ainda, agravaram o quadro daquelas pessoas com algum diagnóstico prévio de transtorno mental.

Alguns estudos demonstram diversas mudanças emocionais nos indivíduos que adotaram o distanciamento social, como relatos de depressão, estresse, humor depressivo, irritabilidade, insônia e sintomas de estresse pós-traumático (FARO A, et al., 2020; LEI L, et al., 2020). Estudos realizados na China evidenciaram elevada prevalência de depressão (50\%) nesse período, sobretudo na classe trabalhadora do setor de saúde. Sabe-se que fatores frequentemente encontrados, como as condições difíceis de trabalho e a falta de reconhecimento profissional atingem os profissionais de saúde, em especial, os profissionais de enfermagem, o que somado ao contexto de pandemia contribui para o aumento de transtornos depressivos nessa população (HUANG Y e ZHAO N, 2020; BARROS MBA, et al., 2020).

Ainda, uma pesquisa realizada no formato on-line no período de maio a abril de 2020 no Brasil constatou que o sentimento frequente de tristeza/depressão foi relatado por $40 \%$ dos adultos brasileiros, enquanto a sensação de ansiedade/nervosismo foi referida como frequente por mais de $50 \%$ dos entrevistados. Dentre essa população, os adultos jovens (faixa de 18 a 29 anos), foram os mais afetados em relação aos transtornos citados, visto que esta faixa etária tem estilo de vida bastante ativo, com desempenho de diferentes atividades pessoais e profissionais, sentindo dessa forma, os efeitos do isolamento de maneira mais intensa (BARROS MBA, et al., 2020).

O mesmo estudo também reporta que a prevalência dos sentimentos de tristeza esteve mais elevada entre as mulheres e pessoas com diagnóstico prévio de depressão (BARROS MBA, et al., 2020). Além disso, um estudo realizado em abril de 2020 na Etiópia, descreveu um aumento de três vezes no predomínio de sintomas de depressão na população participante em comparação com as estimativas anteriores ao contexto de pandemia (UNITED NATIONS, 2020).

Dessa forma, é possível compreender que o contexto pandêmico causou impactos psíquicos em diferentes populações do mundo, de diferentes contextos e faixas etárias, contribuindo para que, principalmente, as taxas de transtornos depressivos se elevaram. Com o agravamento psíquico da população devido ao contexto vivenciado, é fundamental que a assistência em saúde mental não seja posicionada em segundo plano. Como limitação deste estudo cita-se a dificuldade de generalizar os resultados, pois a amostra é limitada a uma Unidade de Atenção Psicossocial de um Hospital Universitário do interior do Rio Grande do Sul. 


\section{CONCLUSÃO}

Os resultados desta pesquisa demonstram que a pandemia causada pelo coronavírus impacta nos serviços de saúde mental, uma vez que medidas necessárias para enfrentar a situação de emergência, como o cancelamento e interrupção de alguns serviços acabaram por influenciar no funcionamento destes. Além disso, verificou-se que mais mulheres internaram nos períodos analisados, o que se deve, principalmente, às questões estruturais, questões de gênero e condições socioeconômicas que permeiam a sociedade. Com relação aos diagnósticos das internações, tem-se um número maior de pessoas com transtornos depressivos, o que reflete os impactos do distanciamento social e a diminuição de contato físico, somado a fatores que permeiam a condição biopsicossocial/financeira das pessoas.

\section{REFERÊNCIAS}

1. AMARANTE P, NUNES MO. Psychiatric reform in the SUS and the struggle for a society without asylums. Revista Ciência e Saúde Coletiva, 2018; 23(6): 2067-74.

2. BARROS MBA, et al. Relato de tristeza/depressão, nervosismo/ansiedade e problemas de sono na população adulta brasileira durante a pandemia de COVID-19. Revista Epidemiologia e Serviços de Saúde, 2020; 29(4): e2020427.

3. BRAGÉ EG, et al. Perfil de internações psiquiátricas femininas: uma análise crítica. Jornal Brasileiro de Psiquiatria, 2020; 69(3): 165-70.

4. BRASIL. MINISTÉRIO DA SAÚDE. Cadernos de Atenção Básica: Saúde Mental. 2013. Disponível em: https://bvsms.saude.gov.br/bvs/publicacoes/cadernos_atencao_basica_34_saude_mental.pdf. Acessado em 23 de Julho de 2021.

5. BRASIL. MINISTÉRIO DA SAÚDE. Lei № 10.216, de 6 de abril de 2001. Brasília: Ministério da Saúde; 2001. Disponível em: https://hpm.org.br/wp-content/uploads/2014/09/lei-no-10.216-de-6-de-abril-de-2001.pdf. Acessado em: 12 de novembro de 2020.

6. BRASIL. Secretaria da Saúde (ES). Diretrizes Clínicas em Saúde Mental. Disponível em: https://saude.es.gov.br/Media/sesa/Protocolo/Diretrizes\%20Clinicas\%20em\%20saude\%20mental.pdf Acessado em 23 de julho de 2021.

7. BRASIL. Secretaria da Saúde (MS). Manual de condutas para enfrentamento do COVID-19. 2020a. Disponível em: https://www.saude.ms.gov.br/wp-content/uploads/2020/04/Manual-de-Condutas-corrigido-15.04.2020.pdf. Acessado em: 28 de janeiro de 2021.

8. BRASIL. Secretaria da Saúde (RS). Orientações aos serviços da rede de atenção psicossocial sobre estratégias de prevenção de disseminação do COVID-19. 2020b. Disponível em: https://saudeadmin.rs.gov.br/upload/arquivos/202004/01120813-orientacoes-raps-covid19-31-03-2020.pdf Acessado em: 27 de janeiro de 2021.

9. BRASIL. Secretaria da Saúde (RS). Plano Estadual de Saúde: 2016/2019. Grupo de Trabalho de Planejamento, Monitoramento e Avaliação da Gestão. 2016. Disponível em: https://saude.rs.gov.br/upload/arquivos/201701/05153251-pes-2016-2019-sesrs.pdf. Acessado em: 18 de fevereiro de 2021.

10. BRASIL. Telessaúde RS. Qual a diferença de distanciamento social, isolamento e quarentena?. 2020c. Disponível em: https://www.ufrgs.br/telessauders/posts_coronavirus/qual-a-diferenca-de-distanciamento-social-isolamento-equarentena/. Acessado em: 18 de fevereiro de 2021.

11. DUARTE MQ, et al. Covid-19 and the impacts on mental health: a sample from Rio Grande do Sul, Brazil. Revista Ciência e Saúde Coletiva, 2020; 25 (9): 3401-11.

12. EMPRESA BRASILEIRA DE SERVIÇOS HOSPITALARES (EBSERH). Plano de contingência para infecção humana pelo novo coronavírus (COVID-19). 2020.2 Disponível em: http://www2.ebserh.gov.br/documents/219273/1462926/Plano+de+Conting\%C3\%AAncia+HUSM+e+anexos++vers\%C3\%A30+4.pdf/a40d3a6d-fba8-4700-a140-29efd9dfadbd. Acessado em: 20 de fevereiro de 2021.

13. FARO A, et al. COVID-19 e saúde mental: a emergência do cuidado. Estudos de Psicologia. Campinas, 2020; 37: e200074.

14. FERREIRA OGL, et al. Active aging and its relationship to functional Independence. Revista Texto e Contexto Enfermagem, 2012; 21 (3): 513-8.

15. HUANG Y, ZHAO N. Generalized anxiety disorder, depressive symptoms and sleep quality during COVID-19 outbreak in China: a web-based cross-sectional survey. Journal of Psychiatric Research, 2020; 288:112954.

16. LA REGINA M, et al. 2020. In: Recomendações de segurança dos pacientes para a epidemia de covid-19: Lições da Experiência Italiana. $2020 . \quad$ Disponível em: https://isqua.org/images/RECOMENDA\%C3\%87\%C3\%95ES_DE_SEGURAN\%C3\%87A_DOS_PACIENTES_PARA A_EPIDEMIA_DE_COVID-19_V1.1.pdf. Acessado em: 20 de janeiro de 2021.

17. LEI L, et al. Comparison of Prevalence and Associated Factors of Anxiety and Depression Among People Affected by versus People Unaffected by Quarantine During the COVID-19 Epidemic in Southwestern China. Medical Science Monitor, 2020; 26: e924609. 
18. MARTIN PS, MEUCCI RD. Epidemiologia dos Transtornos Mentais Comuns entre mulheres na zona rural de Rio Grande, RS, Brasil. Revista Ciência e Saúde Coletiva, 2020; 25 (8): 3087-95.

19. NORONHA KVMS, et al. The COVID-19 pandemic in Brazil: analysis of supply and demand of hospital and ICU beds and mechanical ventilators under different scenarios. Cadernos de Saúde Pública, 2020; 36 (6): e00115320.

20. ORNELL F, et al. "Pandemic fear" and COVID-19: mental health burden and strategies. Brazilian Journal of Psychiatry, 2020; 42 (3): 232-35.

21. PEREIRA AR, JOAZEIRO G. Percepção da internação em hospital psiquiátrico por pacientes com diagnóstico de esquizofrenia. Revista O Mundo da Saúde, 2015; 39 (4): 476-83.

22. PEREIRA MD, et al. A pandemia de COVID-19, o isolamento social, consequências na saúde mental e estratégias de enfrentamento: uma revisão integrativa. Research, Society and Development Journal, 2020; 9 (7): e652974548.

23. QIU J, et al. A nationwide survey of psychological distress among Chinese people in the COVID-19 epidemic: implications and policy recommendations. General psychiatry, 2020; 33 (1): e1002130.

24. SCHMIDT B, et al. Saúde mental e intervenções psicológicas diante da pandemia do novo coronavírus (COVID-19). Estudos de Psicologia, 2020; 37: e200063.

25. UNITED NATIONS. Policy Brief: COVID-19 and the Need for Action on Mental Health. 2020. Disponível em: https://www.un.org/sites/un2.un.org/files/un_policy_brief-covid_and_mental_health_final.pdf. Acessado em: 23 de julho de 2021.

26. ZANARDO GLP, et al. Dispositivos e conexões da rede de atenção psicossocial (RAPS) de Porto Alegre - RS. Est. Inter. Psicol. 2018; 9(3):80-101.

27. ZANARDO GLP, et al. Psychiatric admission and readmission in a general hospital of Porto Alegre: sociodemographic, clinic, and use of Network for Psychosocial Care characteristics. Revista Brasileira de Epidemiologia, $2017 ; 20$ (3): 460-474.

28. ZANELLO V, et al. Saúde mental e gênero: facetas gendradas do sofrimento psíquico. Fractal: Revista de Psicologia, $2015 ; 27$ (3): 238-46. 